\title{
Instabilities in asymmetric nuclear matter
}

\author{
S.S. Avancini, ${ }^{1}$ L. Brito, ${ }^{2}$ D.P.Menezes, ${ }^{1}$ and C. Providência ${ }^{2}$ \\ ${ }^{1}$ Depto de Física - CFM - Universidade Federal de Santa Catarina - Florianópolis - SC - CP. 476 - CEP 88.040 - 900 - Brazil \\ ${ }^{2}$ Centro de Física Teórica - Depto de Fúsica - Universidade de Coimbra - 3000 - Portugal
}

\begin{abstract}
The existence of phase transitions from liquid to gas phases in asymmetric nuclear matter (ANM) is related with the instability regions which are limited by the spinodals. In this work we investigate the instabilities in ANM described within relativistic mean field hadron models, both with constant and density dependent couplings at zero and finite temperatures. In calculating the proton and neutron chemical potentials we have used an expansion in terms of Bessel functions that is convenient at low densities. The role of the isovector scalar $\delta$-meson is also investigated in the framework of relativistic mean field models and density dependent hadronic models. It is shown that the main differences occur at finite temperature and large isospin asymmetry close to the boundary of the instability regions.
\end{abstract}

PACS number(s): 21.65.+f, 21.90.+f, 24.10.Jv, 21.30.Fe

\section{INTRODUCTION}

Recently there has been a big development in the description of nuclei and nuclear matter in terms of relativistic many body theory. In particular, the phenomenological models developed using the relativistic mean field theory describe well the ground state of both stable and unstable nuclei [1, 2]. These same models, with conveniently adjusted parameters, are used to describe the properties of neutron stars and supernovae [3, 4]. Therefore, it is important to test these models at finite temperature and different densities.

The discussion of the properties of asymmetric nuclear matter (ANM) systems, namely their instabilities and phase transitions, are presently a topic of great insterest [5, 6, 7, 8, 9, 10, 11]. The instabilities present in ANM may manifest themselves as an isospin distillation or fractionation [12]. It has recently been discussed [8, 10] that in an ANM system not only the mechanical but also the chemical instabilities appear as an instability of the system against isoscalar fluctuations. Hence the spinodal instability is dominated by density fluctuations which lead to a liquid gas separation with restoration of the isospin symmetry in the dense phase. This is known as the fractionation effect. Multifragmentation also takes place when the system enters the spinodal region through nucleation or through spinodal decomposition. The correlation between spinodal decomposition and negative heat capacity evidences the fact that the spinodal decomposition is the dynamics underlying the liquid gas phase transition [13]. In the afore mentioned work, the authors point out the fact that systematic measurements including correlations between these signals and also the inclusion of the isospin degree of freedom are important sources of future experimental work.

On the other hand it is expected that neutron stars have a solid crust formed by non uniform neutron rich matter in $\beta$-equilibrium above a liquid mantle. In the inner crust, nuclei which form a lattice to reduce the Coulomb energy, coexist with a gas of neutrons which have dripped out. The solid crust has an important role in explaining the sudden spin jumps known as glitches in neutron stars 14]. Recently it has been proposed that there is a relationship between the neutron skin of heavy nuclei and the properties of neutron star crusts [4], namely the thicker the neutron skin of a heavy nucleus the thinner the solid crust of a neutron star. Properties of the crust, namely its thickness and pressure at the crust-core interface, depend largely on the density dependence of the equation of state (EOS). It is of particular importance the transition density below which uniform neutron rich matter becomes unstable. In [4] it was also shown that the thicker the neutron skin the lower the transition density form a uniform to a non uniform neutron rich matter.

Within the framework of relativistic models, the liquid gas phase transition in nuclear matter has been investigated at zero and finite temperatures for symmetric and asymmetric infinite systems [5, 15, 16, 17. With the help of the Thomas - Fermi approximation, we have investigated droplet formation in the liquid gas phase transition in cold [18] and hot 19] asymmetric nuclear matters using relativistic mean field (RMF) models [1, 20]. In [21] we have considered a droplet immersed in a gas of evaporated particles, in such a way that they mimic a source of changing mass. As temperature increases particles evaporate, mainly neutrons, and the fraction of protons in the droplet increases leading to isospin fractionation. In [22] we have shown that at finite temperature droplet properties within different parametrizations of the RMF model have different behaviors with temperature.

The EOS of neutron rich matter is in particular sensitive to the density dependence of the symmetry energy. In [4] the effect of changing this dependence was studied through the inclusion of non linear $\sigma-\rho$ and $\omega-\rho$ couplings. 
On the other hand the authors of 23] claim that the isovector scalar meson $\delta$ is of vital importance in finding the stability conditions of drip-line exotic nuclei because the structure of relativistic interactions with a scalar (attractive) and a vector (repulsive) potential, which balance each other, is also present in the isovector channel. The $\delta$-field has also important effects on the symmetry properties of the nuclear system.

Standard RMF interactions have their limitations even for describing nuclei close to the stablility line. This is due to the fact that the isovector channel is poorly constrained by experimental data. An example is the systematic overestimate of the neutron skins [24]. Some of these limitations are overcome by quantum hadrodynamical models with density dependent meson-nucleon couplings (which we refer to as TW) [25, 26, 27], which have been used with success to describe both nuclear matter and finite nuclei. In these models the couplings are either taken from Dirac-Brueckner-Hartree-Fock calculations or are fitted to data of nuclear matter or finite nuclei.

We may ask whether the recent improvements of the RMF models, both through the inclusion of density dependent meson-nucleon couplings and/or the $\delta$ scalar-isovector meson, present different features at subnuclear densities of nuclear asymmetric matter which could have consequences for the properties of the inner crust of neutron stars or in multifragmentation or isospin fractionation reactions. The parametrizations of these models take generally into account saturation properties of nuclear matter and properties of stable nuclei. Extension of the model for very asymmetric nuclear matter or to finite temperatures may show different behaviors. The simplest test between the models is a comparison of the regions of uniform unstable matter.

In the present work we direct out investigation to some of the topics we have briefly mentioned before. We study the liquid gas phase transition and, in particular, determine the instability regions occuring within the density dependent hadronic models [25, 28] and the non linear relativistic mean field models. We also study models including the $\delta$ meson.

In order to determine the instability regions, we found convenient to generalize the calculation of the chemical potentials as prescribed in [29] to relativisitc models with density dependent effective masses. The expansion we present in our paper is accurate in the range of temperatures and densities discussed and allows us to perform explicit derivatives on the chemical potentials.

This paper is organized as follows: in section 2 we present the whole formalism in which our calculations are based, namely the relativistic models, the chemical potential expansions and the thermodynamical conditions for the description of the instability regions; in section 3 a brief explanation regarding the introduction of the isovector-scalar meson is reported; in section 4 the results are presented and the conclusions are drawn. Finally we include an appendix with some important formulae not given in the main text.

\section{THE FORMALISM}

We start from the lagrangian density of the relativistic TW model 25]

$$
\begin{gathered}
\mathcal{L}=\bar{\psi}\left[\gamma_{\mu}\left(i \partial^{\mu}-\Gamma_{v} V^{\mu}-\frac{\Gamma_{\rho}}{2} \vec{\tau} \cdot \vec{b}^{\mu}\right)-\left(M-\Gamma_{s} \phi\right)\right] \psi \\
+\frac{1}{2}\left(\partial_{\mu} \phi \partial^{\mu} \phi-m_{s}^{2} \phi^{2}\right)-\frac{1}{4} \Omega_{\mu \nu} \Omega^{\mu \nu}+\frac{1}{2} m_{v}^{2} V_{\mu} V^{\mu}-\frac{1}{4} \vec{B}_{\mu \nu} \cdot \vec{B}^{\mu \nu}+\frac{1}{2} m_{\rho}^{2} \vec{b}_{\mu} \cdot \vec{b}^{\mu},
\end{gathered}
$$

where $\Omega_{\mu \nu}=\partial_{\mu} V_{\nu}-\partial_{\nu} V_{\mu}, \vec{B}_{\mu \nu}=\partial_{\mu} \vec{b}_{\nu}-\partial_{\nu} \vec{b}_{\mu}-\Gamma_{\rho}\left(\vec{b}_{\mu} \times \vec{b}_{\nu}\right)$. The parameters of the model are: the nucleon mass $M=939 \mathrm{MeV}$, the masses of the mesons $m_{s}, m_{v}, m_{\rho}$, and the density dependent coupling constants $\Gamma_{s}$, $\Gamma_{v}$ and $\Gamma_{\rho}$, which are adjusted in order to reproduce some of the nuclear matter bulk properties, using the following parametrization:

$$
\Gamma_{i}(\rho)=\Gamma_{i}\left(\rho_{\text {sat }}\right) f_{i}(x), \quad i=s, v
$$

with

$$
f_{i}(x)=a_{i} \frac{1+b_{i}\left(x+d_{i}\right)^{2}}{1+c_{i}\left(x+d_{i}\right)^{2}}
$$

where $x=\rho / \rho_{\text {sat }}$ and

$$
\Gamma_{\rho}(\rho)=\Gamma_{\rho}\left(\rho_{\text {sat }}\right) \exp \left[-a_{\rho}(x-1)\right]
$$


with the values of the parameters $m_{i}, \Gamma_{i}, a_{i}, b_{i}, c_{i}$ and $d_{i}, i=s, v, \rho$ given in [25]. Other possibilities for these parameters are also found in the literature [30]. Notice that in this model the non-linear terms are not present, in contrast with the usual non-linear Walecka model (NLWM).

Within the Thomas-Fermi approximation, the thermodynamic potential is obtained. After it is minimized with respect to the meson fields, the following equations are obtained

$$
\begin{gathered}
\phi_{0}=\frac{\Gamma_{s}}{m_{s}^{2}} \rho_{s}, \\
V_{0}=\frac{\Gamma_{v}}{m_{v}^{2}} \rho, \\
b_{0}=\frac{\Gamma_{\rho}}{2 m_{\rho}^{2}} \rho_{3},
\end{gathered}
$$

with

$$
\begin{gathered}
\rho=\rho_{p}+\rho_{n}, \quad \rho_{3}=\rho_{p}-\rho_{n}, \quad \rho_{i}=2 \int \frac{\mathrm{d}^{3} p}{(2 \pi)^{3}}\left(f_{i+}-f_{i-}\right), \quad i=p, n \\
\rho_{s}=\rho_{s p}+\rho_{s n}, \quad \rho_{s i}=2 \int \frac{\mathrm{d}^{3} p}{(2 \pi)^{3}} \frac{M^{*}}{E^{*}}\left(f_{i+}+f_{i-}\right),
\end{gathered}
$$

$M^{*}=M-\Gamma_{s} \phi_{0}, E^{*}=\sqrt{\mathbf{p}^{2}+M^{* 2}}$ and $f_{i \pm}=1 /\left\{1+\exp \left[\left(E^{*} \mp \nu_{i}\right) / T\right]\right\}$, where the effective chemical potential is

$$
\nu_{i}=\mu_{i}-\Gamma_{v} V_{0}-\tau_{i 3} \frac{\Gamma_{\rho}}{2} b_{0}-\Sigma_{0}^{R}, \quad \tau_{p 3}=1, \tau_{n 3}=-1
$$

with the rearrangement term given by

$$
\Sigma_{0}^{R}=\frac{\partial \Gamma_{v}}{\partial \rho} \rho V_{0}+\frac{\partial \Gamma_{\rho}}{\partial \rho} \rho_{3} \frac{b_{0}}{2}-\frac{\partial \Gamma_{s}}{\partial \rho} \rho_{s} \phi_{0}
$$

The energy density in the mean field approximation reads:

$$
\begin{aligned}
\mathcal{E}\left(\Gamma_{s}, \Gamma_{v}, \Gamma_{\rho}\right)= & 2 \sum_{i} \int \frac{\mathrm{d}^{3} p}{(2 \pi)^{3}} E^{*}\left(f_{i+}+f_{i-}\right)+ \\
& \frac{m_{s}^{2}}{2} \phi_{0}^{2}+\frac{m_{v}^{2}}{2} V_{0}^{2}+\frac{m_{\rho}^{2}}{2} b_{0}^{2}
\end{aligned}
$$

The pressure becomes

$$
\begin{aligned}
P\left(\Gamma_{s}, \Gamma_{v}, \Gamma_{\rho}\right) & =\frac{1}{3 \pi^{2}} \sum_{i} \int \mathrm{d} p \frac{\mathbf{p}^{4}}{E^{*}}\left(f_{i+}+f_{i-}\right)-\frac{m_{s}^{2}}{2} \phi_{0}^{2}\left(1+2 \frac{\rho}{\Gamma_{s}} \frac{\partial \Gamma_{s}}{\partial \rho}\right) \\
& +\frac{m_{v}^{2}}{2} V_{0}^{2}\left(1+2 \frac{\rho}{\Gamma_{v}} \frac{\partial \Gamma_{v}}{\partial \rho}\right)+\frac{m_{\rho}^{2}}{2} b_{0}^{2}\left(1+2 \frac{\rho}{\Gamma_{\rho}} \frac{\partial \Gamma_{\rho}}{\partial \rho}\right)
\end{aligned}
$$

For two of the usual NLWM parametrizations, namely NL3 [31] and TM1 [32], the above equations read:

$$
\mathcal{E}_{N L 3}=\mathcal{E}\left(g_{s}, g_{v}, g_{\rho}\right)+\frac{\kappa \phi_{0}^{3}}{6}+\frac{\lambda \phi_{0}^{4}}{24}, \quad P_{N L 3}=P\left(g_{s}, g_{v}, g_{\rho}\right)-\frac{\kappa \phi_{0}^{3}}{6}-\frac{\lambda \phi_{0}^{4}}{24}
$$

and

$$
\mathcal{E}_{T M 1}=\mathcal{E}\left(g_{s}, g_{v}, g_{\rho}\right)+\frac{\kappa \phi_{0}^{3}}{6}+\frac{\lambda \phi_{0}^{4}}{24}+\frac{\xi g_{v}^{4} V_{0}^{4}}{8}, P_{T M 1}=P\left(g_{s}, g_{v}, g_{\rho}\right)-\frac{\kappa \phi_{0}^{3}}{6}-\frac{\lambda \phi_{0}^{4}}{24}+\frac{\xi g_{v}^{4} V_{0}^{4}}{24}
$$


where the meson-nucleon coupling constants, $g_{s}, g_{v}$, and $g_{\rho}$ substitute $\Gamma_{s}, \Gamma_{v}$, and $\Gamma_{\rho}$. They are not density dependent and consequently all derivative terms in the pressure cancel out and $\kappa, \lambda$ and $\xi$ are the self-coupling constants multiplying the non-linear terms.

In order to study the instability region at low densities it is convenient to invert eq. (8) and obtain the effective chemical potential that appear inside the distribution functions. We have followed the prescription given in [6, 29], where just the particle distribution function is considered. The effective chemical potential reads:

$$
\nu_{i}=\frac{1}{\beta}\left(\ln \left(\eta_{i}\right)+\sum_{l=1}^{\infty} b_{l} \eta_{i}^{l}\right), \quad i=p, n
$$

where

$$
\eta_{i}=\frac{\rho_{i}}{\gamma Q\left(\beta M^{*}\right)}
$$

$\gamma$ is the spin multiplicity, $\beta=1 / T$ and

$$
Q\left(\beta M^{*}\right)=\int \frac{d^{3} p}{(2 \pi)^{3}} e^{-\beta E^{*}}=\frac{M^{* 2}}{2 \pi^{2} \beta} K_{2}\left(\beta M^{*}\right),
$$

with $K_{n}$ the modified Bessel function. The coefficients $b_{l}$ are defined in terms of the ratios

$$
S_{n}=\frac{Q\left(n \beta M^{*}\right)}{Q\left(\beta M^{*}\right)}=\frac{K_{2}\left(n \beta M^{*}\right)}{n K_{2}\left(\beta M^{*}\right)},
$$

and have been calculated in [29]. We list the first three

$$
b_{1}=a_{2}, \quad b_{2}=a_{3}-a_{2}^{2} / 2, \quad b_{3}=a_{4}-a_{2} a_{3}+a_{2}^{3} / 3,
$$

with $a_{2}=S_{2}\left(\beta M^{*}\right), a_{3}=2 S_{2}^{2}\left(\beta M^{*}\right)-S_{3}\left(\beta M^{*}\right), a_{4}=5 S_{2}^{3}\left(\beta M^{*}\right)-5 S_{3}\left(\beta M^{*}\right) S_{2}\left(\beta M^{*}\right)+S_{4}\left(\beta M^{*}\right)$. The chemical potentials $\mu_{i}, i=p, n$ are obtained from equation (9) and they read

$$
\mu_{i}=\Gamma_{v} V_{0}+\tau_{i 3} \frac{\Gamma_{\rho}}{2} b_{0}+\Sigma_{0}^{R}+\frac{1}{\beta}\left(\ln \left(\eta_{i}\right)+\sum_{l=1}^{\infty} b_{l} \eta_{i}^{l}\right)
$$

where the rearrangement term is given in equation (10).

We have checked the range of applicability of the expansion given in eq.15 by comparing it with the exact results. We have concluded that, for symmetric matter, the expansion works very well for temperatures $T \geq 7 \mathrm{MeV}$ and subsaturation densities, $\rho<\rho_{0}$. This agreement improves for higher temperatures within the range of temperatures involved in the present work, where the antiparticles do not play a crucial role. For ANM, $T=7 \mathrm{MeV}$, the agreement is still good for the proton chemical potential. The neutron chemical potential is well reproduced only for $\rho<0.8 \rho_{0}$ as can be seen in fig. 11. where we have plotted the effective chemical potentials for protons and neutrons for asymmetric matter with the proton fraction $y_{p}=0.1$, where $y_{p}=\frac{\rho_{p}}{\rho}$. This result is still adequate for the range of densities we have considered in the present work

The stability conditions for asymmetric nuclear matter, keeping volume and temperature constant, are obtained from the free energy density $\mathcal{F}$, imposing that this function is a convex function of the densities $\rho_{p}$ and $\rho_{n}$, i.e. the symmetric matrix with the elements [5, 7, 10]

$$
\mathcal{F}_{i j}=\left(\frac{\partial^{2} \mathcal{F}}{\partial \rho_{i} \partial \rho_{j}}\right)_{T},
$$

is positive. This is equivalent to imposing [33]

$$
\begin{gathered}
\frac{\partial \mu_{p}}{\partial \rho_{p}}>0, \\
\frac{\partial\left(\mu_{p}, \mu_{n}\right)}{\partial\left(\rho_{p}, \rho_{n}\right)}>0,
\end{gathered}
$$


where we have used $\mu_{i}=\left.\frac{\partial \mathcal{F}}{\partial \rho_{i}}\right|_{T, \rho_{j \neq i}}$. In terms of the proton fraction the conditions (18) and (19) can be rewritten, respectively, in the form

$$
\left(\frac{\partial P}{\partial \rho}\right)_{T, y_{p}}>0
$$

and

$$
\left(\frac{\partial P}{\partial \rho}\right)_{T, y_{p}}\left(\frac{\partial \mu_{p}}{\partial y_{p}}\right)_{T, P}>0
$$

It has recently been argued [8, 10] that in ANM the spinodal instabilities cannot be separately classified as mechanical or chemical instabilities. In fact, the two conditions that give rise to the instability of the system are coupled so that it appears as a mixture of baryon density and concentration fluctuations. Therefore, we define the stability region as determined by (20).

In order to calculate the boundaries of the spinodal instability regions we use the Gibbs-Duhem relation, at a fixed temperature and isospin asymmetry,

$$
\left(\frac{\partial P}{\partial \rho}\right)_{T, \delta}=\frac{\rho}{2}\left[(1+\delta) \frac{\partial \mu_{n}}{\partial \rho}+(1-\delta) \frac{\partial \mu_{p}}{\partial \rho}\right]=\sum_{i=p, n} \rho_{i}\left(\frac{\partial \mu_{i}}{\partial \rho}\right)_{\delta}
$$

with $\delta=-\rho_{3} / \rho=1-2 y_{p}$, and

$$
\left(\frac{\partial \mu}{\partial \delta}\right)_{T, P}=\left(\frac{\partial \mu}{\partial \delta}\right)_{T, \rho}-\left(\frac{\partial \mu}{\partial \rho}\right)_{T, \delta}\left(\frac{\partial P}{\partial \rho}\right)_{T, \delta}^{-1}\left(\frac{\partial P}{\partial \delta}\right)_{T, \rho},
$$

where

$$
\left(\frac{\partial P}{\partial \delta}\right)_{T, \rho}=\frac{\rho}{2}\left[(1+\delta) \frac{\partial \mu_{n}}{\partial \delta}+(1-\delta) \frac{\partial \mu_{p}}{\partial \delta}\right]=\sum_{i=p, n} \rho_{i}\left(\frac{\partial \mu_{i}}{\partial \delta}\right)_{\rho} .
$$

With the expressions given in this section the spinodal regions can be obtained for both different temperatures and different parametrizations.

\section{INCLUDING ISOVECTOR-SCALAR MESONS}

To investigate the influence of the $\delta$-meson in the stability conditions we have included in the NLWM the isovectorscalar meson terms 23]:

$$
\begin{gathered}
\mathcal{L}=\bar{\psi}\left[\gamma_{\mu}\left(i \partial^{\mu}-g_{v} V^{\mu}-\frac{g_{\rho}}{2} \vec{\tau} \cdot \vec{b}^{\mu}\right)-\left(M-g_{s} \phi-g_{\delta} \vec{\tau} \cdot \vec{\delta}\right)\right] \psi \\
+\frac{1}{2}\left(\partial_{\mu} \phi \partial^{\mu} \phi-m_{s}^{2} \phi^{2}-\frac{1}{3} \kappa \phi^{3}-\frac{1}{12} \lambda \phi^{4}\right)-\frac{1}{4} \Omega_{\mu \nu} \Omega^{\mu \nu}+\frac{1}{2} m_{v}^{2} V_{\mu} V^{\mu} \\
-\frac{1}{4} \vec{B}_{\mu \nu} \cdot \vec{B}^{\mu \nu}+\frac{1}{2} m_{\rho}^{2} \vec{b}_{\mu} \cdot \vec{b}^{\mu}+\frac{1}{2}\left(\partial_{\mu} \vec{\delta} \partial^{\mu} \vec{\delta}-m_{\delta}^{2} \vec{\delta}^{2}\right)
\end{gathered}
$$

where $g_{\delta}$ and $m_{\delta}$ are respectively the coupling constant of the $\delta$ meson with the nucleons and its mass. Self-interacting terms for the $\sigma$-meson are also included, $\kappa$ and $\lambda$ denoting the corresponding coupling constants. The set of constants is defined by $g_{i}=\sqrt{f_{i} m_{i}^{2}}, i=s, v, \delta, \frac{g_{\rho}}{2}=\sqrt{f_{\rho} m_{\rho}^{2}}, m_{s}=550 \mathrm{MeV}, m_{v}=783 \mathrm{MeV}, m_{\rho}=763 \mathrm{MeV}, m_{\delta}=980 \mathrm{MeV}$, $f_{s}=10.33 \mathrm{fm}^{2}, f_{v}=5.42 \mathrm{fm}^{2}, f_{\rho}=3.15 \mathrm{fm}^{2}, f_{\delta}=2.5 \mathrm{fm}^{2}, \kappa=0.066 g_{s}^{3}$ and $\lambda=-6 \times 0.0048 g_{s}^{4}[23]$ and we call it NL $\delta$. For reference, in Table \we show the properties of nuclear matter reproduced by the models we discuss in the present work. From the minimization of the thermodynamic potential obtained in a Thomas-Fermi approach, the equation of motion for this field becomes

$$
\delta_{3}=\frac{g_{\delta}}{m_{\delta}^{2}} \rho_{s 3}
$$


with $\rho_{s 3}=\rho_{s p}-\rho_{s n}$. The energy density and the pressure are also affected by the presence of the new meson. The term $+1 / 2 m_{\delta}^{2} \delta_{3}^{2}$ should be added to the energy density and the $-1 / 2 m_{\delta}^{2} \delta_{3}^{2}$ should be added to the expression of the pressure, both given in eq. (13). The effective masses for protons and neutrons acquire different values, namely,

$$
M_{i}^{*}=M-g_{s} \phi_{0}-\tau_{i 3} g_{\delta} \delta_{3} \quad i=p, n .
$$

For completeness, we have also included the $\delta$-meson in a model where the $\rho$ and $\delta$ couplings are density dependent, as done in [28], where it is called density dependent hadronic model $(\mathrm{DDH} \rho \delta)$. For this purpose, we have considered the density dependence of the $\rho$ and $\delta$-nucleon vertices given in fig. 1 of ref. 28] which have been extracted from DBHF calculations of ref. 34]. In this case the coupling constants $g_{s}, g_{v}, g_{\rho}$ and $g_{\delta}$ used in equation (24) should be replaced by $\Gamma_{s}, \Gamma_{v}, \Gamma_{\rho}$ and $\Gamma_{\delta}$ and the non-linear scalar terms do not appear. For $\Gamma_{s}$ and $\Gamma_{v}$ we take the parametrizations given in Eqs.(2) and (4). For $\Gamma_{\rho}$ and $\Gamma_{\delta}$, we propose the following parametrization,

$$
\Gamma_{i}=\Gamma\left(\rho_{\text {sat }}\right) f_{i}(x), \quad x=\rho / \rho_{\text {sat }}
$$

with

$$
f(x)=a_{i} \exp \left[-b_{i}(x-1)\right]-c_{i}\left(x-d_{i}\right), \quad i=\rho, \delta .
$$

and the parameters $a_{i}, b_{i}, c_{i}$ and $d_{i}$ are defined in Table This parametrization reproduces the curves given in fig. 1 of [28] and is also displayed in fig. 2 of the present work. We point out that in the present work there is a factor 2 difference in the definition of $\rho$-meson coupling constant. We take for the effective chemical potential Eq. (9), with the rearrangement term given by

$$
\Sigma_{0}^{R}=\frac{\partial \Gamma_{v}}{\partial \rho} \rho V_{0}+\frac{\partial \Gamma_{\rho}}{\partial \rho} \rho_{3} \frac{b_{0}}{2}-\frac{\partial \Gamma_{s}}{\partial \rho} \rho_{s} \phi_{0}-\frac{\partial \Gamma_{\delta}}{\partial \rho} \rho_{s 3} \delta_{3}
$$

and for the pressure

$$
P\left(\Gamma_{s}, \Gamma_{v}, \Gamma_{\rho}, \Gamma_{\delta}\right)=P\left(\Gamma_{s}, \Gamma_{v}, \Gamma_{\rho}\right)-\frac{m_{\delta}^{2}}{2} \delta_{3}^{2}\left(1+2 \frac{\rho}{\Gamma_{\delta}} \frac{\partial \Gamma_{\delta}}{\partial \rho}\right)
$$

\section{RESULTS AND CONCLUSIONS}

A quantity of interest in ANM is the nuclear bulk symmetry energy discussed in [23]. This quantity is important in studies involving neutron stars and radioactive nuclei. The behavior of the symmetry energy at densities larger than nuclear saturation density is still not well established. In general, relativistic and non-relativistic models give different predictions for the symmetry energy.

It is usually defined as

$$
\mathcal{E}_{\text {sym }}=\left.\frac{1}{2} \frac{\partial^{2} \mathcal{E} / \rho}{\partial \delta^{2}}\right|_{\delta=0},
$$

which, for the models not including the $\delta$-meson, can be analytically rewritten as

$$
\mathcal{E}_{\text {sym }}=\frac{k_{F}^{2}}{6 E_{F}^{*}}+\frac{\Gamma_{\rho}^{2}}{8 m_{\rho}^{2}} \rho,
$$

where

$$
k_{F p}=k_{F}(1+\delta)^{1 / 3}, \quad k_{F n}=k_{F}(1-\delta)^{1 / 3},
$$

with $k_{F}=\left(1.5 \pi^{2} \rho\right)^{1 / 3}$. If the $\delta$ meson is included the symmetry energy reads $[23]$ :

$$
\mathcal{E}_{\text {sym }}=\frac{k_{F}^{2}}{6 E_{F}^{*}}+\frac{\rho}{2}\left[\frac{\Gamma_{\rho}^{2}}{4 m_{\rho}^{2}}-\frac{\Gamma_{\delta}^{2}}{m_{\delta}^{2}}\left(\frac{M^{*}}{E_{F}^{*}}\right)^{2}\right] .
$$

In fig 3 we show the symmetry energy for the different models used in this work, calculated at $T=0$ and $y_{p}=0.5$. We can see that at subsaturation densities where the instability regions occur ( 0 up to $\left.0.1 \mathrm{fm}^{-3}\right)$, NL3 and TM1 
parametrizations give very similar behaviours. The NL $\delta$ parametrization describes saturation at $0.16 \mathrm{fm}^{-3}$ and this fact affects the symmetry energy at low densities, in particular it is the model with the lowest symmetry energy in this range of densities (see Table【). The TW model presents a different behaviour, after a faster increase at low densities, the symmetry energy increases much slower than most of the other models at larger densities. A very low value of the symmetry energy was obtained with the $\mathrm{DDH} \rho \delta$ parametrization. It amounts to $25 \mathrm{MeV}$, in contrast with the value mentioned in 28] $(33.4 \mathrm{MeV})$. Nevertheless, in the same reference [28], the authors show a curve in fig. 2, which confirms our number. The density dependent hadronic models show a softer symmetry energy at the densities shown. At higher densities the models containing the $\delta$-meson are expected to get a harder behaviour due to relativistic effects, namely the $\delta$ contribution goes to zero and only the repulsive contribution from the $\rho$-meson remains [23]. We point out that in present work we are only testing the low density region of the symmetry energy, namely our discussion is concerned with the behaviour of nuclear matter at densities below $0.7 \rho_{0}$, where $\rho_{0}$ is the saturation density. In this region the quantities which better distinguish the different models are the slope $L=\left.3 \rho_{0}\left(\frac{\partial \mathcal{E}_{s y m}}{\partial \rho}\right)\right|_{\rho=\rho_{0}}$ and curvature $K_{\text {sym }}=\left.9 \rho_{0}^{2}\left(\frac{\partial^{2} \mathcal{E}_{\text {sym }}}{\partial \rho^{2}}\right)\right|_{\rho=\rho_{0}}$ of the symmetry energy [35] given in Table \. The TW model is the only one with a negative curvature at saturation density. In fact, the $\mathrm{DDH} \rho \delta$ also has a negative curvature at densites below 0.13 $\mathrm{fm}^{-3}$ but at saturation the curvature has already a positive value.

In fig 4 we plot the spinodals for the NL3 parameter set and different temperatures, varying from $\mathrm{T}=0$ up to $\mathrm{T}=14$ $\mathrm{MeV}$, already close to the critical temperature, $T_{N L 3, c} \sim 15 \mathrm{MeV}$. All the other models present a similar behaviour, namely the larger the temperature the smaller the instability region. These same conclusions have already been discussed in [6] for Skyrme type phenomenologial EOS for ANM. After a critical temperature the liquid-gas phase transition is a smooth transition. A consequence of this behavior is the greater stability against density flucutations of neutron-rich systems. Also, in intermediate energy collisions fragmentation or fractionation occurs later after the system has cooled down.

In fig. [5 we display the spinodals for different parameter sets obtained with $T=0,10,14 \mathrm{MeV}$. For $T=0 \mathrm{MeV}$ the differences are not significant, occuring at the higher density branch, $\sim 0.1 \mathrm{fm}^{-3}$. In particular, in the NL $\delta$ parametrization the boundary lies at a larger density than in the other models for $\delta<0.5$. The spinodals for the density dependent hadronic models are decreasing less with the asymmetry parameter $\delta$ than the others. At finite temperature the differences are larger. These differences occur again in the larger density branch, and it is again the NL $\delta$ which presents a boundary at larger densities in the small asymmetry region followed by the TW and the $\mathrm{DDH} \rho \delta$ models. In the large asymmetry region we are already testing both the critical temperatures and asymmetries of the models and the differences between the models are larger. For the $\mathrm{DDH} \rho \delta$ model, as temperature increases, the instability boundaries extend to higher asymmetries as compared with all the other models. This is possibly due to a lower symmetry energy. The opposite occurs with the NL3 parametrization which has the larger symmetry energy. We conclude that the information obtained from the spinodal decomposition sensitive to the underlying model comes from the phase space close to the spinodal boundary at large isospin asymmetry.

In conclusion, we have compared the spinodal boundary as a function of density and isospin asymmetry, at several temperatures, for different relativistic models. We have considered both quantum hadrodynamical approaches with non-linear terms (NL3, TM1, NL $\delta$ ) and density dependent hadronic models with density dependent coupling parameters $(\mathrm{TW}, \mathrm{DDH} \rho \delta)$. The largest differences between the models occur at finite temperature and are more clearly shown in the high isospin asymmetry region. Only physical quantities that explore this region in phase space, as for example the neutron-proton differential flow suggested in [36], will bring possible information about the EOS of ANM. Another physical system that could be sensitive to present results is a protoneutron star in the process of stellar colapse. The physical structure of the matter, namely a smaller or larger non-uniform nuclear matter region, may affect properties such as the neutrino scattering processes [37].

Another point to be investigated is the role of the Coulomb interaction and finite size effects in the instabilities of ANM. We have already shown that the electromagnetic force cannot be neglected in nucleation processes, where droplets of lower asymmetry are formed in a very asymmetric gas background either at $T=0$ or finite temperatures [18, 19]. The authors of ref. [9] have included the Coulomb interaction and surface tension in their calculation of the binodals in a simplified way and they have shown that the minimum pressure for a given temperature do not occur for symmetric nuclear matter and that surface effects lower the binodal pressure. It has also been shown that the Coulomb interaction affects the growth of instabilities [38]. A natural extension of the present work would be the inclusion of the Coulomb interaction in the same spirit as done in $[\underline{9}$ and consequent construction of the binodal sections and spinodal regions for different temperatures. We expect, however, that the overall effect of the inclusion of the Coulomb interaction will be the same for all the models and the main conclusions of the present work will not change. 


\section{APPENDIX: SOME FORMULAE}

In what follows $T$ is considered fixed. We give the main expressions needed to obtain the conditions which define the boundaries of the instability regions. We first consider the derivatives of the chemical potential with respect to $\rho$, for a given $\delta$. For this purpose we need the derivative of the effective chemical potential that reads:

$$
\left(\frac{\partial \nu_{i}}{\partial \rho}\right)_{\delta}=\frac{\partial \nu_{i}}{\partial \eta_{i}} \frac{\partial \eta_{i}}{\partial \rho}+\frac{1}{\beta} \sum_{k} \eta_{i}^{k} \frac{d b_{k}}{d M^{*}} \frac{\partial M^{*}}{\partial \rho}
$$

where

$$
\frac{\partial \nu_{i}}{\partial \eta_{i}}=\frac{1}{\beta}\left[\frac{1}{\eta_{i}}+\sum_{k} k b_{k} \eta_{i}^{k-1}\right]
$$

and

$$
\frac{\partial \eta_{i}}{\partial \rho}=\frac{\eta_{i}}{\rho}-\frac{\beta^{4} \pi^{2} \rho_{i}}{2}\left[\frac{4 K_{2}(x)-x\left(K_{1}(x)+K_{3}(x)\right)}{x^{3} K_{2}^{2}(x)}\right] \frac{\partial M^{*}}{\partial \rho}, \quad x=\beta M^{*} .
$$

So, in the NLWM model, one has

$$
\left(\frac{\partial \mu_{i}}{\partial \rho}\right)_{\delta}=\left(\frac{\partial \nu_{i}}{\partial \rho}\right)_{\delta}+\frac{g_{v}^{2}}{m_{v}^{2}}-\tau_{i 3} \frac{g_{\rho}^{2}}{4 m_{\rho}^{2}} \delta
$$

Instead, for the TW model, the previous equation reads

$$
\left(\frac{\partial \mu_{i}}{\partial \rho}\right)_{\delta}=\left(\frac{\partial \nu_{i}}{\partial \rho}\right)_{\delta}+\frac{\partial}{\partial \rho}\left(\frac{\Gamma_{v}^{2}}{m_{v}^{2}} \rho\right)-\tau_{i 3} \frac{\partial}{\partial \rho}\left(\frac{\Gamma_{\rho}^{2}}{4 m_{\rho}^{2}} \delta \rho\right)+\frac{\partial \Sigma_{0}^{R}}{\partial \rho}
$$

with

$$
\begin{gathered}
\Sigma_{0}^{R}=\frac{\Gamma_{v}}{m_{v}^{2}} \frac{\partial \Gamma_{v}}{\partial \rho} \rho^{2}+\frac{\Gamma_{\rho}}{4 m_{\rho}^{2}} \frac{\partial \Gamma_{\rho}}{\partial \rho} \rho^{2} \delta^{2}-\frac{m_{s}^{2}}{\Gamma_{s}^{3}} \frac{\partial \Gamma_{s}}{\partial \rho}\left(M-M^{*}\right)^{2} \\
M-M^{*}=\left(\frac{\Gamma_{s}}{m_{s}}\right)^{2} \rho_{s} .
\end{gathered}
$$

To evaluate the expressions involved in (22) we obtain the derivative of the chemical potential with respect to $\delta$ for a fixed $\rho$

$$
\left(\frac{\partial \nu_{i}}{\partial \delta}\right)_{\rho}=\frac{\partial \nu_{i}}{\partial \eta_{i}} \frac{\partial \eta_{i}}{\partial \delta}+\frac{1}{\beta} \sum_{k} \eta_{i}^{k} \frac{d b_{k}}{d M^{*}} \frac{\partial M^{*}}{\partial \delta}
$$

where

$$
\frac{\partial \eta_{i}}{\partial \delta}=-\frac{\tau_{3 i} \rho}{2 \gamma Q(x)}-\frac{\beta^{4} \pi^{2} \rho_{i}}{2}\left[\frac{4 K_{2}(x)-x\left(K_{1}(x)+K_{3}(x)\right)}{x^{3} K_{2}^{2}(x)}\right] \frac{\partial M^{*}}{\partial \delta} .
$$

Both the derivatives $\frac{\partial M^{*}}{\partial \rho}$ and $\frac{\partial M^{*}}{\partial \delta}$ are calculated numerically.

The expressions for $\left(\frac{\partial \mu_{i}}{\partial \delta}\right)_{\rho}$ become

$$
\left(\frac{\partial \mu_{i}}{\partial \delta}\right)_{\rho}=\left(\frac{\partial \nu_{i}}{\partial \delta}\right)_{\rho}-\tau_{3 i}\left(\frac{g_{\rho}}{2 m_{\rho}}\right)^{2} \rho
$$


in the NLWM, and

$$
\left(\frac{\partial \mu_{i}}{\partial \delta}\right)_{\rho}=\left(\frac{\partial \nu_{i}}{\partial \delta}\right)_{\rho}-\tau_{3 i}\left(\frac{\Gamma_{\rho}}{2 m_{\rho}}\right)^{2} \rho+\frac{\Gamma_{\rho}}{2 m_{\rho}^{2}} \frac{\partial \Gamma_{\rho}}{\partial \rho} \rho^{2} \delta+\frac{\partial \Sigma_{0}^{R}}{\partial \delta}
$$

in the TW model.

Some derivatives of the coefficients $b_{l}$ are also listed:

$$
\begin{gathered}
\frac{d b_{1}}{d M^{*}}=\beta \frac{d S_{2}}{d x} \\
\frac{d b_{2}}{d M^{*}}=\beta \frac{d b_{2}}{d x}=\beta\left(3 S_{2} \frac{d S_{2}}{d x}-\frac{d S_{3}}{d x}\right),
\end{gathered}
$$

with

$$
\frac{d S_{n}(x)}{d x}=\frac{1}{2 n K_{2}^{2}(x)}\left[K_{2}(n x)\left(K_{1}(x)+K_{3}(x)\right)-n K_{2}(x)\left(K_{1}(n x)+K_{3}(n x)\right)\right]
$$

\section{ACKNOWLEDGMENTS}

This work was partially supported by CNPq (Brazil), CAPES(Brazil)/GRICES (Portugal) under project 100/03 and FEDER/FCT (Portugal) under the project POCTI /35308/ FIS/ 2000.

[1] P.-G. Reinhard, M. Rufa, J. Maruhn, W. Greiner and J. Friedrich, Z. Phys. A 323, 13 (1986).

[2] Y. Sugahara and H. Toki, Nucl. Phys. A579, 557 (1994).

[3] H. Shen, H. Toki, K. Oyamatsu and K. Sumiyoshi, Nucl. Phys. A637, 435 (1998); K. Sumiyoshi and H. Toki, Astrophys. J. 422, 700 (1994); D.P. Menezes and C. Providência, Phys. Rev. C 68, 035804 (2003); P.K. Panda, D.P. Menezes and C. Providência, Phys. Rev. C 69025207 (2004); D.P. Menezes and C. Providência, nucl-th/0312050 Phys. Rev. C (in press).

[4] C. J. Horowitz and J. Piekarewicz, Phys. Rev. Lett. 86, 5647 (2001)

[5] H. Müller and B.D. Serot, Phys. Rev. C 52, 2072 (1995).

[6] B.-A. Li, A. T. Sustich, M. Tilley and B. Zhang, Nucl. Phys. A699, 493 (2002).

[7] V. Baran, M. Colonna, M. Di Toro, and A. B. Larionov, Nucl. Phys. A632, 287 (1998).

[8] V. Baran, M. Colonna, M. Di Toro, and V. Greco, Phys. Rev. Lett. 86, 4492 (2001).

[9] S. J. Lee and A. Z. Mekjian, Phys. Rev. C 63, 044605 (2001).

[10] J. Margueron and P. Chomaz, Phys. Rev. C 67, 041602 (2003);P. Chomaz, M. Colonna and J. Randrup, Phys. Rep. 389, $263(2004)$.

[11] S. J. Lee and A. Z. Mekjian, Phys. Rev. C 68, 014608 (2003).

[12] H. S. Xu, et al, Phys. Rev. Lett. 85, 716 (2000).

[13] B. Borderie et al., nucl-exp/0311016

[14] P. W. Anderson and N. Itoh, Nature (London) 256, 25 (1975), D. Pines and M. A. Alpar, Nature (London) 316, 27 (1985); B. Link, R. I. Epstein and J. M. Lattimer, Phys. Rev. Lett. 83, 3362 (1999).

[15] H. Reinhardt and H. Schulz, Nucl. Phys. A432, 630 (1985).

[16] H. Müller and R.M. Dreizler, Nucl. Phys. A563, 649 (1993).

[17] D. Von Eiff, J. M. Pearson, W. Stocker and M. K.Weigel, Phys. Lett. B 324, 279 (1994).

[18] D.P. Menezes and C. Providência, Nucl. Phys. A650, 283 (1999); G. Krein, D. P. Menezes, M. Nielsen and C. Providência, Nucl. Phys. A674, 125 (2000).

[19] D.P. Menezes and C. Providência, Phys. Rev. C 60, 024313 (1999).

[20] J. Boguta and A. R. Bodmer, Nucl. Phys. A292, 413 (1977); A.R. Bodmer and C.E. Price, Nucl. Phys. A505, 123 (1989); A.R. Bodmer, Nucl. Phys. A526, 703 (1991).

[21] D. P. Menezes and C. Providência, Phys. Rev. C 64, 044306 (2001).

[22] C Providência, D. P. Menezes and L. Brito, Nucl. Phys. A703, 188 (2002).

[23] B. Liu, V. Greco, V. Baran, M. Colonna and M. Di Toro, Phys. Rev. C 65, 045201 (2002).

[24] B. Alex Brown, Phys. Rev. Lett. 85, 5296 (2000).

[25] S. Typel and H. H. Wolter, Nucl. Phys. A656, 331 (1999).

[26] T. Niksic, D. Vretenar and P. Ring, Phys. Rev. C 66, 064302 (2002).

[27] S.S. Avancini, M.E. Bracco, M. Chiapparini and D.P. Menezes, Phys. Rev C 67, 024301 (2003); S.S. Avancini, M.E. Bracco, M. Chiapparini and D.P. Menezes, J. Phys. G 30, 27 (2004). 
[28] T. Gaitanos, M. Di Toro, S. Typel, V. Baran, C. Fuchs, V. Greco and H. H. Wolter, Nucl. Phys. A 732, 24 (2004).

[29] H. R. Jaqaman, A. Z. Mekjian, and L. Zamick, Phys. Rev. C 29, 2067 (1984).

[30] G. Hua, L.Bo and M. Di Toro, Phys. Rev. C 62, 035203 (2000).

[31] G. A. Lalazissis, J. König and P. Ring, Phys. Rev. C 55, 540 (1997).

[32] K. Sumiyoshi, H. Kuwabara, H. Toki, Nucl. Phys. A 581, 725 (1995).

[33] L. D. Landau and E. M. Lifshitz, Statistical Physics, vol I, (Pergamon Press, New York, 1989), p. 288.

[34] F. de Jong, H. Lenske, Phys. Rev. C 57, 3099 (1998).

[35] B.-A. Li, C. M. Ko and W. Bauer, Int. J. Mod. Phys. E 7 (1997) 147.

[36] Bao-An Li, Phys. Rev. Lett. 85, 4221 (2000)

[37] D. G. Ravenhall, C. J. Pethick and J. R. Wilson, Phys. Rev. Lett. 50, 2066 (1983); C. J. Horowitz, M. A. Pérez-García, and J. Piekarewicz Phys. Rev. C 69, 045804 (2004).

[38] G. Fabbri and F. Matera, Phys. Rev. C 58, 1345 (1998).

TABLE I: Nuclear matter properties.

\begin{tabular}{|c|c|c|c|c|c|}
\hline & NL3 [31] & TM1 [32] & TW $[25]$ & NL $\delta[23]$ & DDH $\rho \delta[28]$ \\
\hline$B / A(\mathrm{MeV})$ & 16.3 & 16.3 & 16.3 & 16.0 & 16.3 \\
\hline$\rho_{0}\left(\mathrm{fm}^{-3}\right)$ & 0.148 & 0.145 & 0.153 & 0.160 & 0.153 \\
\hline$K(\mathrm{MeV})$ & 272 & 281 & 240 & 240 & 240 \\
\hline $\mathcal{E}_{\text {sym. }}(\mathrm{MeV})$ & 37.4 & 36.9 & 32.0 & 30.5 & 25.1 \\
\hline$M^{*} / M$ & 0.60 & 0.63 & 0.56 & 0.60 & 0.56 \\
\hline$L(\mathrm{MeV})$ & 123. & 117. & 55. & 101. & 44. \\
\hline$K_{\text {sym }}(\mathrm{MeV})$ & 108. & 36 & -124. & 112. & 45. \\
\hline
\end{tabular}

TABLE II: Parameters of the $D D H \rho \delta$ model

\begin{tabular}{cccccc}
\hline $\mathrm{i}$ & $\Gamma_{i}$ & $a_{i}$ & $b_{i}$ & $c_{i}$ & $d_{i}$ \\
\hline$\rho$ & 5.8635 & 0.095268 & 2.171 & 0.05336 & 17.8431 \\
\hline$\delta$ & 7.58963 & 0.01984 & 3.4732 & -0.0908 & -9.811 \\
\hline
\end{tabular}




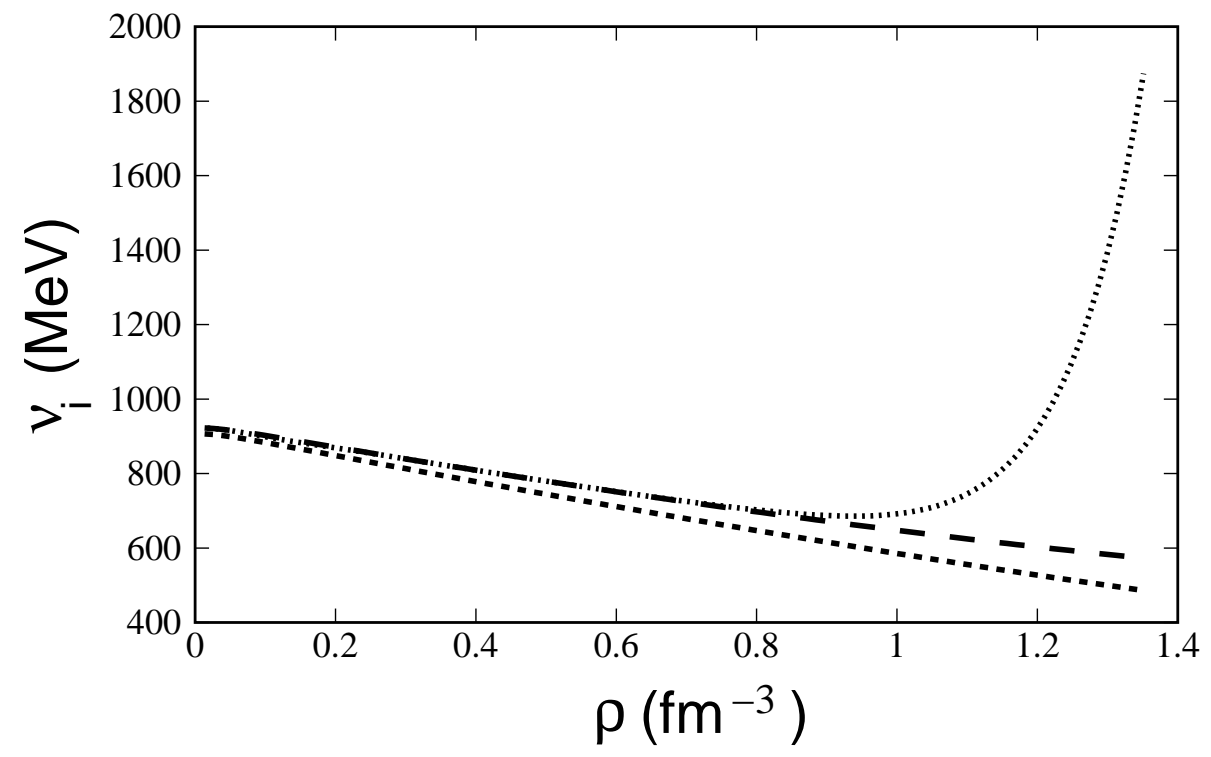

FIG. 1: Chemical potential curves for $T=7 \mathrm{MeV}$, NL3 parameter set and $y_{p}=0.1$. The curves for the exact proton chemical potential and its expansion are coincident (short dashed line). The straight long dashed line represents the exact neutron chemical potential and the bending one (dotted line) its expansion. The expansions are taken up to $5^{\text {th }}$ order.

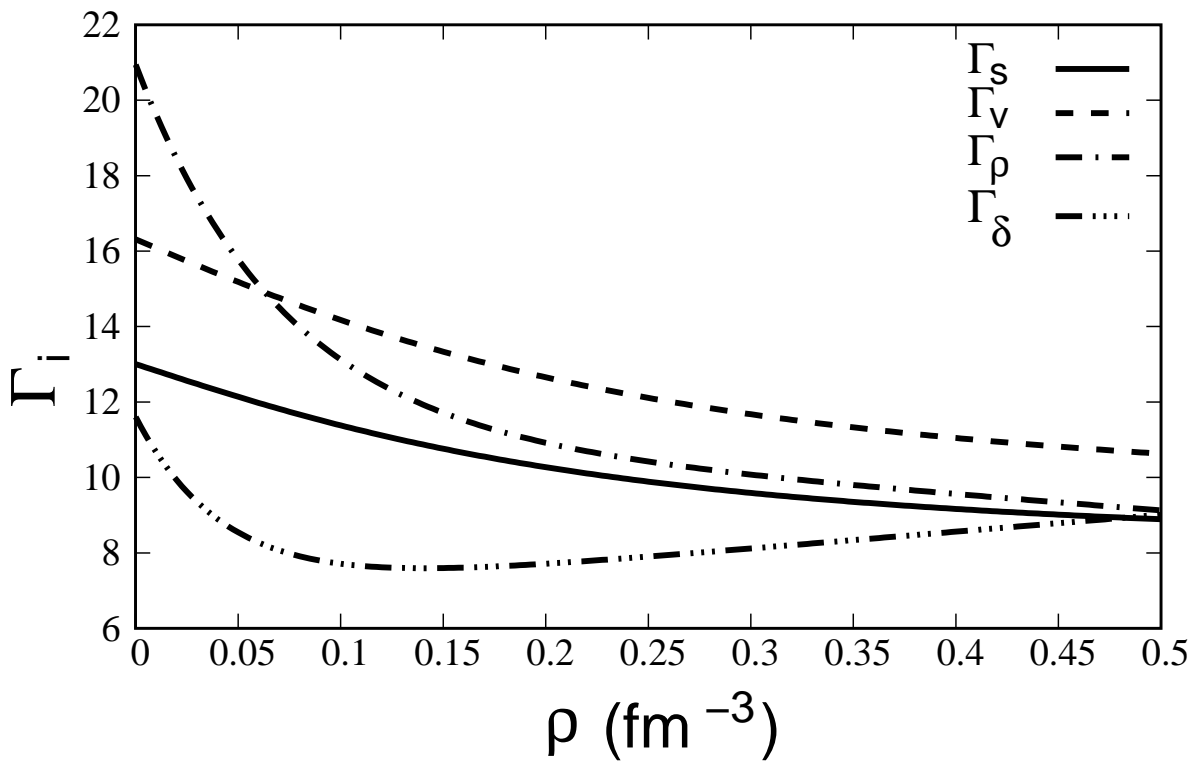

FIG. 2: Density dependent couplings as obtained from the proposed parametrization given in the text and which reproduce the curves given in [28]. 


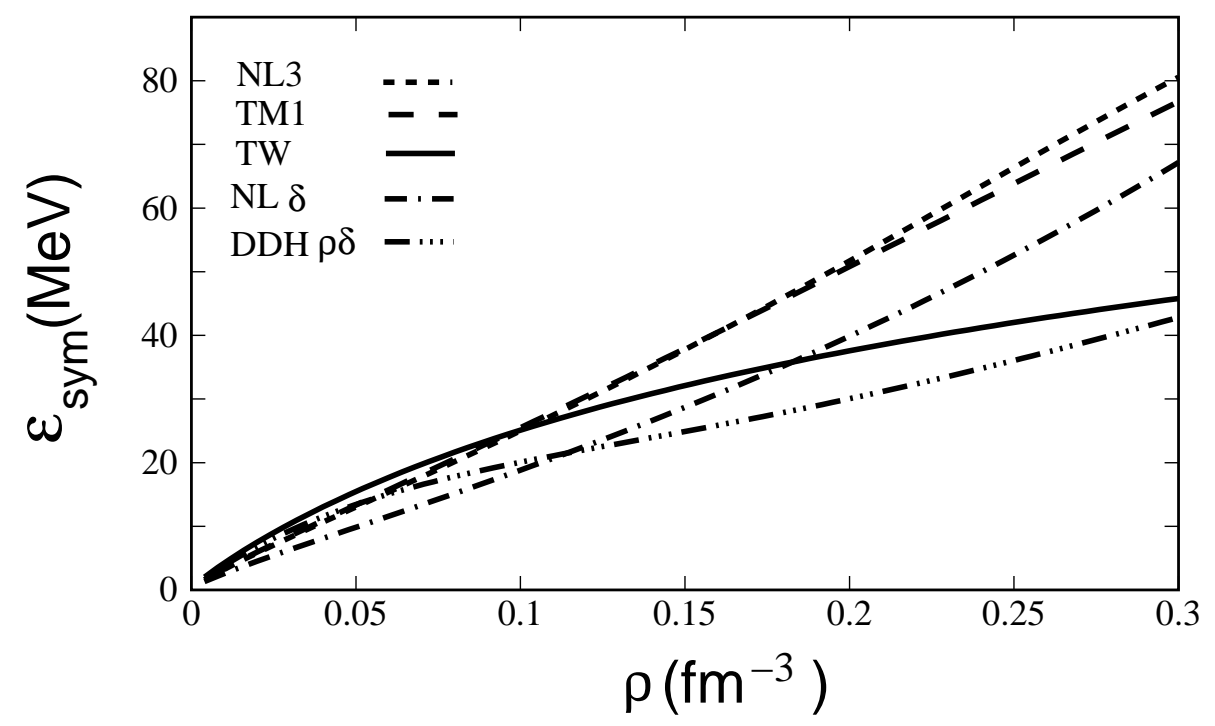

FIG. 3: Symmetry energy results for the NL3, TM1, TW, NL $\delta$ and DDH $\rho \delta$ parameter sets.

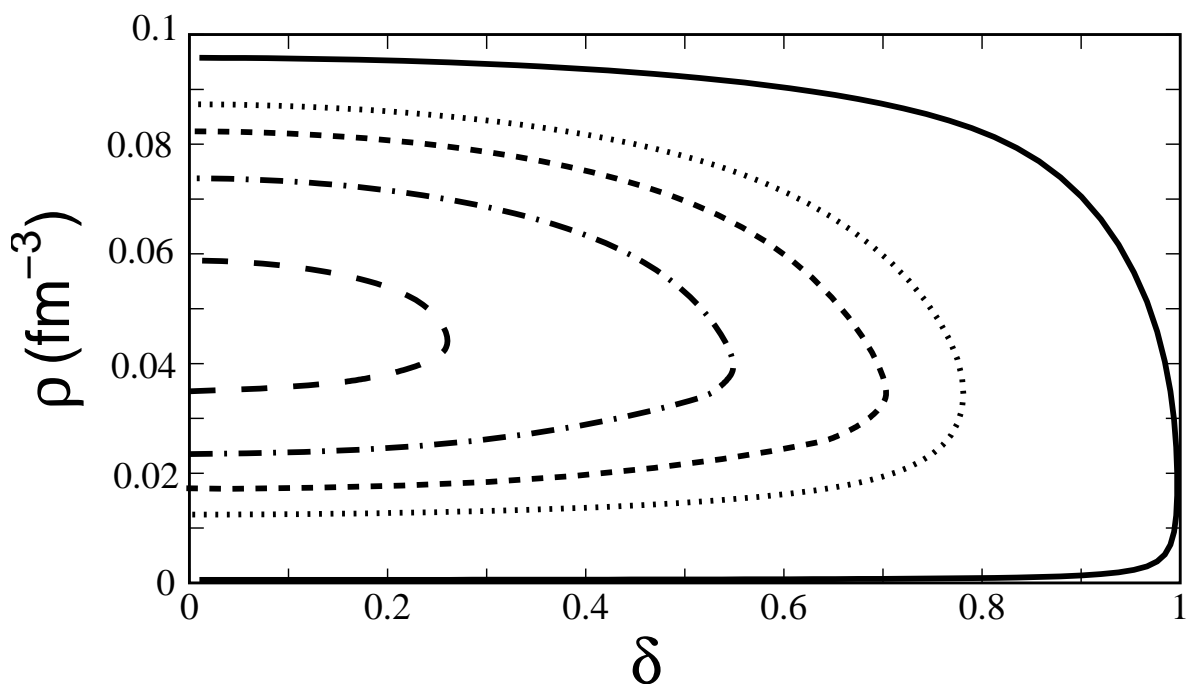

FIG. 4: Spinodal regions obtained with the NL3 parametrization and different temperatures. The instability regions lie inside the curves. The curves are drawn for $\mathrm{T}=0$ (solid line), 8 (dotted line), 10 (short-dashed line), 12 (dash-dotted line) and 14 (long dashed line) $\mathrm{MeV}$. 


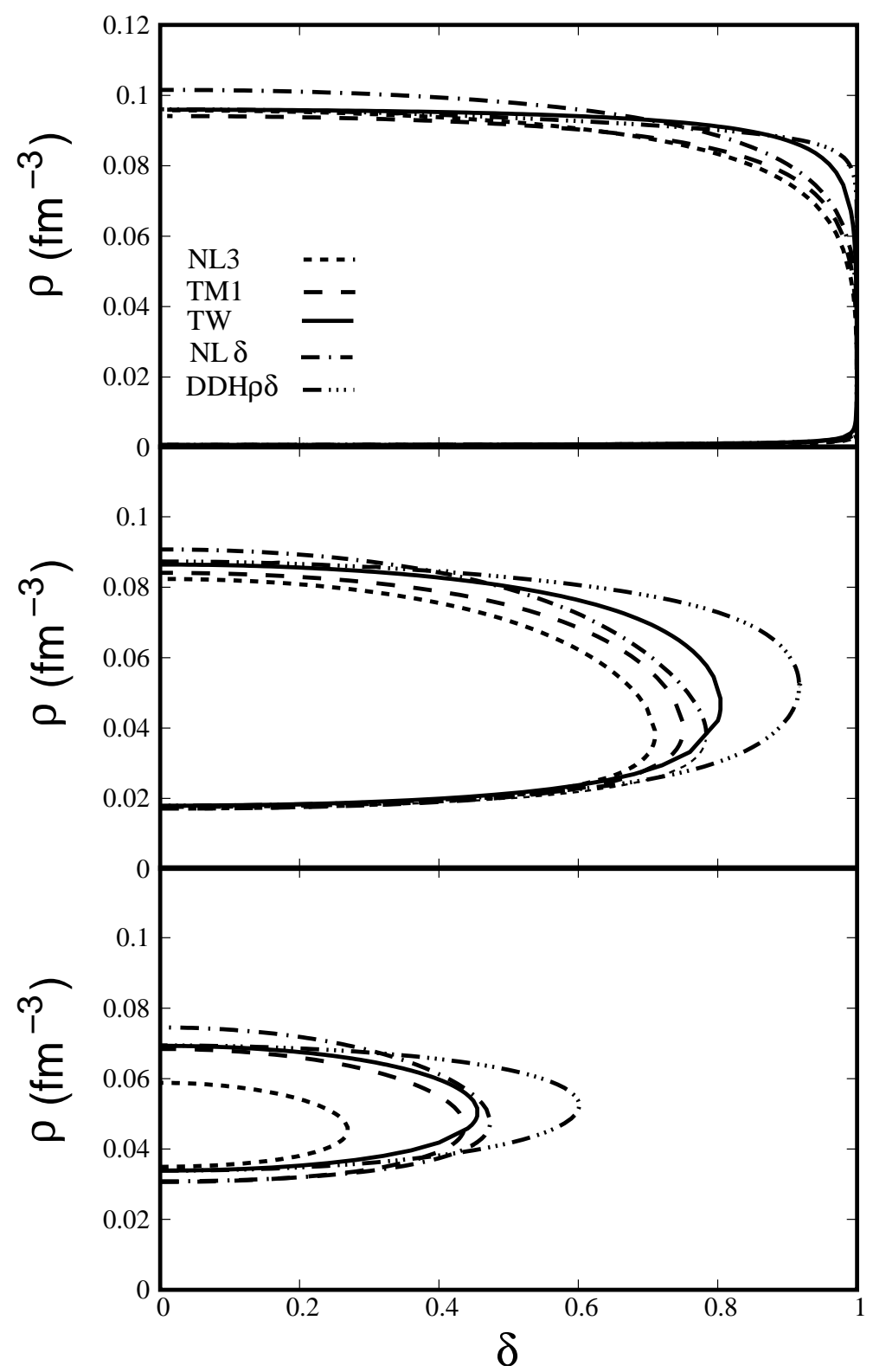

FIG. 5: Spinodal regions for different parameter sets and $T=0$ (upper panel), $T=10 \mathrm{MeV}$ (middle panel) and $T=14 \mathrm{MeV}$ (lower panel). 